\title{
Solar Wind Plasma Particles Organized by the Flow Speed
}

\author{
Viviane Pierrard ${ }^{1,2}($ D $) ~ M a r i a n ~$ Lazar $^{3,4}(D)$ \\ Stepan Štverák ${ }^{5,6}$
}

Received: 2 September 2020 / Accepted: 27 October 2020 / Published online: 5 November 2020

(C) The Author(s) 2020

\begin{abstract}
Recent reports of the first data from Parker Solar Probe (PSP) have pointed to a series of links, correlations or anti-correlations between the solar wind bulk speed $\left(V_{\mathrm{SW}}\right)$ and physical properties of plasma particles from less than $0.25 \mathrm{AU}$ in the corona. In the present paper, we describe corresponding and additional links of solar wind properties, at $0.4 \mathrm{AU}$ and 1.0 AU, in an attempt to complement the PSP data and understand their evolution. A detailed analysis is carried out for the main electron populations, comparing the low-energy (thermal) core and the collisionless suprathermal halo. We show that the anti-correlation observed at $0.4 \mathrm{AU}$ between $V_{\mathrm{SW}}$ and the number density (average value) is maintained also at $1 \mathrm{AU}$ for both the core and halo electrons. On the contrary, only the core electrons manifest a clear anti-correlation of the temperature with $V_{\mathrm{SW}}$, while the halo temperature does not vary much. We also describe the ions, protons and helium, which have a more reduced mobility and their properties exhibit different variations with the solar wind speed. The results are used to shed more light on the mechanisms leading to a differential acceleration of these species and the origin of slow and fast wind modulation.
\end{abstract}

Keywords Solar wind $\cdot$ Electrons $\cdot$ Protons $\cdot$ Observations

\footnotetext{
$凶$ V. Pierrard

viviane.pierrard@aeronomie.be

1 Royal Belgian Institute for Space Aeronomy BIRA-IASB, Space Physics and STCE, 3 av. Circulaire, 1180 Brussels, Belgium

2 Center for Space Radiations (CSR), Georges Lemaître Centre for Earth and Climate Research (TECLIM), Earth and Life Institute (ELI), Université Catholique de Louvain (UCLouvain), Place Louis Pasteur 3 bte L4.03.08, 1348 Louvain-La-Neuve, Belgium

3 Theoretical Physics IV, Ruhr-University Bochum, 44780 Bochum, Germany

4 Centre for Mathematical Plasma Astrophysics, KU Leuven, Celestijnenlaan 200B, 3001 Leuven, Belgium

5 Institute of Atmospheric Physics, Czech Academy of Sciences, Prague, Czech Republic

6 Astronomical Institute, Czech Academy of Sciences, Ondrejov, Czech Republic
} 


\section{Introduction}

Properties of the solar wind (SW) plasma particles have been measured in-situ by a multitude of spacecraft missions, e.g., Ulysses, Helios 1 and 2, Wind, Cluster, etc. (Domingo, 2002; Meyer-Vernet, 2007; Lazar, 2012), enabling also indirect interpretations of plasma physics at low heliocentric distances in the corona where direct in-situ measurements were not possible (Scudder, 1992; Pierrard, Maksimovic, and Lemaire, 2001a,b). The new observations from Parker Solar Probe (PSP) and Solar Orbiter (SO) are expected to confirm these predictions, and explain the main mechanisms which trigger plasma outflows and modulate the main properties of the SW plasma particles, i.e., electrons, protons and heavier ions (Berčič et al., 2020; Halekas et al., 2020; Maksimovic et al., 2020). Measured in-situ, the velocity distributions of different species (subscript s) are used to determine their macroscopic properties (moments), such as number densities $n_{\mathrm{s}}$, bulk velocities $u_{\mathrm{s}}$, temperatures $T_{\mathrm{s}}$, and, implicitly, their plasma betas $\beta_{\mathrm{s}}=8 \pi n_{\mathrm{s}} k_{\mathrm{B}} T_{\mathrm{s}} / B_{0}^{2}$, or temperature anisotropy $T_{\mathrm{s}, \perp} / T_{\mathrm{s}, \|}$, with $\perp$, $\|$ indicating gyrotropic directions with respect to the magnetic field. With increasing distance from the Sun fast wind protons $(\mathrm{s}=\mathrm{p})$ show a clear demagnetization by increasing $\beta_{\mathrm{p}}$, and a quasi-adiabatic switch from a transverse anisotropy $\left(T_{\mathrm{p}, \perp} / T_{\mathrm{p}, \|}>1\right.$ and $\beta_{\mathrm{p}}<1$ at $0.3 \mathrm{AU})$ to one predominant in parallel direction $\left(T_{\mathrm{p}, \perp} / T_{\mathrm{p}, \|}<1\right.$ and $\beta_{\mathrm{p}}>1$ beyond $\left.1 \mathrm{AU}\right)$ (Matteini et al., 2007). Such a clear evolution is not observed in the slow wind, between 0.3 and $1 \mathrm{AU}$ protons are less magnetized accumulating near the equipartition of kinetic and magnetic energy densities $(\beta \approx 1)$ and isotropy $\left(T_{\mathrm{p}, \perp} / T_{\mathrm{p}, \|} \approx 1\right)$, although large departures from this condition are also observed with kind of symmetry in both (gyrotropic) directions, $T_{\mathrm{p}, \perp} / T_{\mathrm{p}, \|} \gtrless 1$ (Stansby et al., 2018). More detailed results (Stansby et al., 2018; Stansby, Horbury, and Matteini, 2019; Ďurovcová, Šafránková, and Němeček, 2019) based on core and beam analysis demonstrate that the core protons are aiming to isotropize towards larger distances, while the impression of increased parallel temperature originates from the beams that become super-Alfvénic (Klein et al., 2019).

By contrast to protons the electrons have a much higher mobility, and their properties may not vary much with the distance from the Sun (Štverák et al., 2008). Their distributions show two central components, a quasi-thermal core at low energies, and high energy tails enhanced by a suprathermal and more diffuse halo (Pierrard, Maksimovic, and Lemaire, 2001a; Štverák et al., 2008; Lazar et al., 2017). Asymmetric field-aligned beams or strahls may also be apparent, but are more specific to fast streams (Maksimovic et al., 2005; Berčič et al., 2019; Wilson et al., 2019). At high radial distances, the number density of the strahl electrons slightly decreases while the halo electron population slightly increases, suggesting that scattering due to some process(es) isotropizes the strahl and enhances the halo (Maksimovic et al., 2005; Štverák et al., 2009). This hypothesis is supported by the increase of strahl angular width with radial distance (Hammond et al., 1996; Berčič et al., 2019), contrary to the decrease predicted by an adiabatic behavior. These characteristics likely indicate the effects of wave-particle interactions and/or Coulomb collisions acting on electron distributions, to reduce their anisotropy but increase their suprathermalization (Vocks et al., 2005; Pierrard, Lazar, and Schlickeiser, 2011). That the halo and the strahl relative densities vary in an opposite sense, as well as the fact that they both lie in the same energy range $\left(10^{2}-10^{3} \mathrm{eV}\right)$, suggests that the halo and strahl electrons may have similar origins.

If the core electrons are still controlled by the binary collisions, preserving a Maxwellianlike distribution, the suprathermal electrons are more dilute and much hotter making them practically collisionless and well reproduced by the so-called Kappa (or $\kappa-$ ) power-law distribution functions (Štverák et al., 2008; Pierrard and Lazar, 2010). The halo temperature increases and the power spectral exponent $\kappa$ decreases with increasing distance from the 
Sun (Pierrard et al., 2016; Lazar et al., 2017). Differences between electron core and halo populations must also be reflected by the variations of their properties with heliospheric distance and the solar wind bulk speed.

Observational studies focus in general on the core electrons (subscript c), due to their dominant number density reaching up to $95 \%$ of total density. The plasma beta and temperature anisotropy of these quasi-thermal electrons do not change much from 0.35 to $2 \mathrm{AU}$, and remain well localized around the equipartition conditions, i.e., $\beta_{\mathrm{c}} \approx 1$ (Štverák et al., 2008). Both the number density and the temperature show a clear anti-correlation with the flow speed in the corona (Halekas et al., 2020) and in the solar wind at low distance, becoming barely noticeable at 1 AU (Maksimovic et al., 2020). These anti-correlations are often invoked to argue that slow or fast winds have different origins in the corona (Gloeckler, Zurbuchen, and Geiss, 2003), despite the very high mobility of electrons, and the fact that proton temperature shows a direct link with the solar wind speed (Elliott et al., 2012). However, this bi-modal organization of the solar wind may need more refined complementary data analysis for understanding and identifying the sources of solar wind (Stansby et al., 2018; Berčič et al., 2020). On the other hand, the suprathermal halo (subscript $h$ ) is more dilute but much hotter than the core (Štverák et al., 2008; Pierrard et al., 2016), making it an important contributor to the total kinetic energy budget, i.e., with $\beta_{\mathrm{h}} \approx \beta_{\mathrm{c}}$. For this reason and due to their antisunward drift, suprathermal electrons are mainly responsible for the heat transport in the solar wind (Štverák, Trávníček, and Hellinger, 2015; Lazar et al., 2020). In a collisionpoor solar wind, there is also observational evidence (Wilson et al., 2013) that suprathermal electrons are (highly) involved in the interaction with plasma waves, being heated by the resonant wave fluctuations (Vocks and Mann, 2003) or stimulating the self-induced electromagnetic fields (Lazar et al., 2019). However, we do not know much about the evolution of halo electrons and their properties with heliocentric distance, or the way these properties correlate with the solar wind speed. Recent reports of PSP data have provided the first radial profiles of electron properties in the corona, within the interval 0.17-0.4 AU, showing a clear anti-correlation of the halo temperature with the solar wind speed (Halekas et al., 2020).

In the present paper, we revisit the main properties of the SW plasma particles, which show similar organization by the flow speed and offer valuable clues on the physical mechanisms involved to reduce this dependence with increasing heliocentric distance. These insights from the existing solar wind data are crucial for building a realistic and unitary picture, in agreement with the advanced observations from new missions, such as PSP and SO. Solar wind models need realistic assumptions concerning the characteristics of the plasma at low distances in the corona to make predictions at larger distances. The velocity distribution function of the particles, as well as their number density, bulk velocity and temperature at low distance have to be assumed to determine the characteristics of the solar wind at any further distance. The possible presence of solar suprathermal electrons in the low corona can heat the corona and accelerate the wind at high bulk velocities (Pierrard and Lemaire, 1996; Maksimovic, Pierrard, and Riley, 1997; Pierrard and Pieters, 2014). To determine the boundary conditions low in the corona, magnetograms observed in the photosphere are generally used to determine the magnetic field intensity at a low distance in the solar corona at a level called the Potential Field Source Surface (PFSS) (Pierrard and Pieters, 2014; Moschou et al., 2017) for instance). From these magnetic field values, boundary conditions are determined using estimated relations (Pierrard and Pieters, 2014; Pomoell and Poedts, 2018) to obtain the plasma characteristics at low radial distance. It is thus crucial to chose the most appropriate values in association with the observations.

We analyze how different characteristics of solar wind plasma particles, electrons, protons and helium, are related to each other at different radial distances in the solar wind, 
Table 1 Solar wind electron data used in the present study (Štverák et al., 2008; Pierrard et al., 2016).

\begin{tabular}{lll}
\hline Dist (AU) & Spacecraft & $\begin{array}{l}\text { Number of } \\
\text { measurements }\end{array}$ \\
\hline $0.35-0.45$ & Helios & 14427 \\
$0.9-1.1$ & Cluster and Helios & 20914 \\
\hline
\end{tabular}

especially (see Table 1) at about $0.4 \mathrm{AU}$ (i.e., between 0.35 and $0.45 \mathrm{AU}$ ), and at about $1 \mathrm{AU}(0.9-1.1 \mathrm{AU})$. Such a study is particularly important to deduce the evolution of the solar wind characteristics. Each species or population of plasma particles, including thermal or suprathermals, e.g., core and halo electrons, may show specific variations with the bulk speed of the solar wind, which can help to understand their origin in the solar corona (e.g., slow winds) or more inner corona (fast winds from coronal holes). This information is encoded in the properties of plasma particles, velocity distributions and moments of the distributions defining macroscopic parameters, which vary continuously with the solar wind expansion in the heliosphere. Understanding these variations may also help to identify key physical processes which overheat the corona and accelerate the solar wind.

\section{Electrons}

The electron data set includes electron core and halo parameters derived from more than 120000 distributions measured in the ecliptic within an extended range of heliocentric distances from 0.29 to 4 AU (Štverák et al., 2008; Pierrard et al., 2016). Štverák et al. (2008) subtracted the field-aligned strahl component and fitted non-drifting part of the observed velocity distribution with a sum of a bi-Maxwellian for the core (subscript c), described by three parameters: $n_{\mathrm{c}}, T_{\mathrm{c}, \|}$ and $T_{\mathrm{c}, \perp}$, and a bi-Kappa for the halo population (subscript $\mathrm{h}$ ) giving other four parameters: $n_{\mathrm{h}}, T_{\mathrm{h}, \|}, T_{\mathrm{h}, \perp}$ and $\kappa$.

Launched respectively in 1974 and 1976, HELIOS 1 and 2 allowed one to identify distinct conditions in (i) a high-speed solar wind $(667 \mathrm{~km} / \mathrm{s}$ in average at $1 \mathrm{AU})$ associated to low densities ( 3 protons $\mathrm{cm}^{-3}$ in average at $1 \mathrm{AU}$ ) and most probably originating from coronal holes, generally located at high latitudes, and (ii) a low-speed solar wind $(320 \mathrm{~km} / \mathrm{s} \mathrm{in}$ average at $1 \mathrm{AU})$, much more variable and associated to higher densities $\left(10\right.$ protons $\mathrm{cm}^{-3}$ in average at 1 AU) (Pierrard and Lemaire, 2001). Launched in 1990, Ulysses showed that at high latitudes, the high-speed solar wind is almost constant during quiet solar activity periods (McComas and et al., 1998). We first analyze the links between electron parameters using data at two different distances in the ecliptic plane: 0.4 AU from HELIOS (measured between 1975 and 1978) and 1 AU from HELIOS and CLUSTER (measured between 2002 and 2003). The data set analyzed here contains also data from larger distances, between 2.7 and 3.3 AU from ULYSSES, but the number of events (1275) is not sufficient to reach clear conclusions, and the links between the parameters tend to disappear with the distance (Pierrard et al., 2016), so that these results are not illustrated here. In the present work we investigate the links that exist between different properties of the plasma particles and their bulk velocity. The solar wind velocity is the first characteristic deduced from the magnetograms, marking different origins of the wind in the solar corona.

\subsection{Electron Core and Halo Densities}

Figures 1 and 2 illustrate the number densities of the core and halo electrons, respectively, as functions of the solar wind bulk velocity. In Figure 1 the velocity ranges between 210 and 

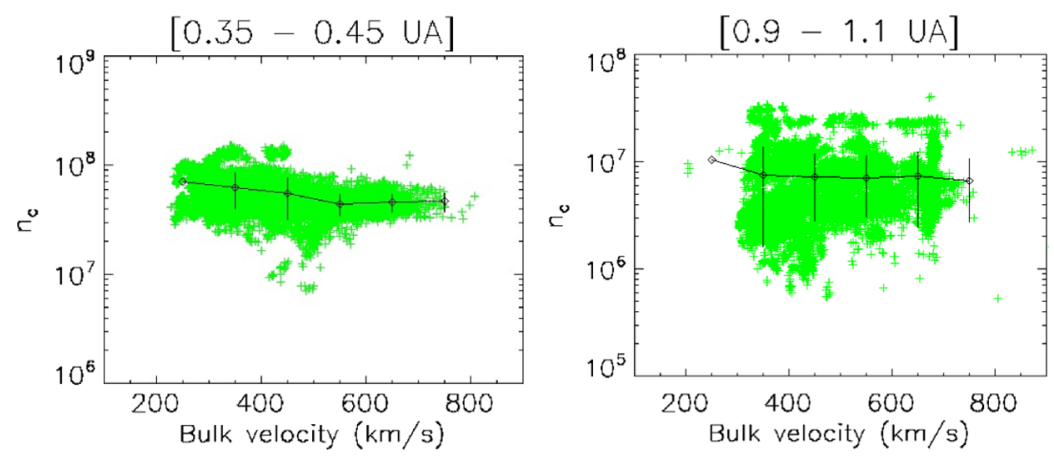

Figure 1 Number density in $\mathrm{m}^{-3}$ of core electrons as a function of the bulk velocity at $0.35-0.45 \mathrm{AU}$ (left) and $0.9-1.1 \mathrm{AU}$ (right).
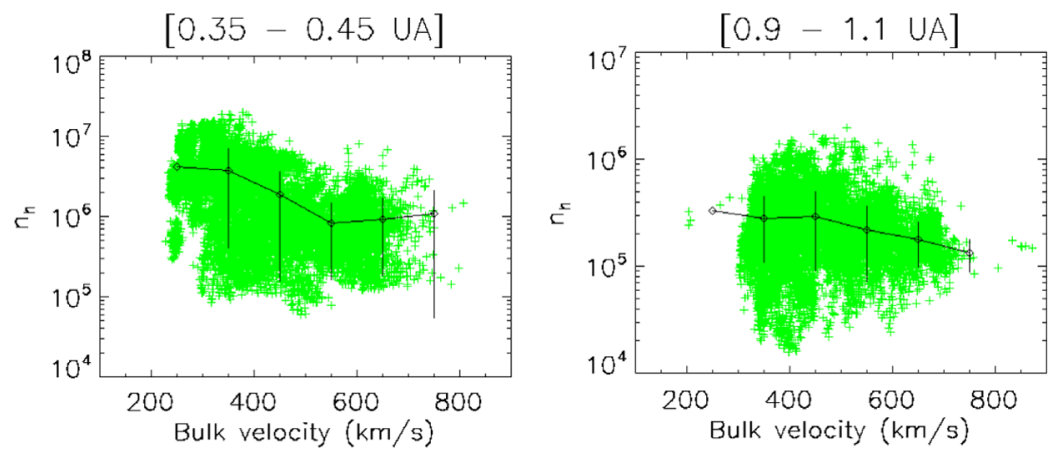

Figure 2 Number density in $\mathrm{m}^{-3}$ of halo electrons as a function of the bulk velocity at $0.35-0.45$ AU (left) and $0.9-1.1 \mathrm{AU}$ (right).

$750 \mathrm{~km} / \mathrm{s}$ at $0.4 \mathrm{AU}$ (left panel), and between 300 and $750 \mathrm{~km} / \mathrm{s}$ at $1 \mathrm{AU}$ (right panel), with only a few observations with higher velocities (up to $950 \mathrm{~km} / \mathrm{s}$ ) probably due to Coronal Mass Ejections (CMEs). The acceleration of the low-speed particles is obvious, but does not affect the fast streams of particles, the maximum limit found at $1 \mathrm{AU}$ is almost the same with that observed at $0.4 \mathrm{AU}$. The mechanisms accelerating particles at lower distances may also operate at higher heliocentric distances, in the interval 0.4-1 AU, but selectively, acting mainly on the low-energy electrons.

At low heliocentric distances, i.e., $\approx 0.4 \mathrm{AU}$, values of the core density are confined in an interval $\left(2 \times 10^{7}<10^{8} \mathrm{~m}^{-3}\right)$ much narrower than that found at $1 \mathrm{AU}$, where the average value of the core density (solid line) is one order of magnitude lower, but the data widely spread exceeding one order of magnitude difference between the minimum and maximum values. This may be a direct consequence of the solar wind expansion combined with a reduction of thermalization of the electron populations (e.g., due to the reduced effect of Coulomb collisions) with increasing the distance from the Sun.

The average values indicate a significant anti-correlation between $n_{\mathrm{c}}$ and $u$ at $0.4 \mathrm{AU}$, with a higher density in the slow solar wind. This result agrees well with the well-known distinct origin of the fast wind in the low density coronal holes. At $1 \mathrm{AU}$ (also at $3 \mathrm{AU}$, not shown here) this anti-correlation is reduced but still apparent, despite the large data dispersion, much larger than at $0.4 \mathrm{AU}$. Either at low or large distances, the number density 
in the slow wind is characterized by a larger spread of values, which strongly suggests that low-speed streams have various origins. Our data are from the ecliptic plane, and may therefore reflect that slow and fast winds interact and tend to reduce these anti-correlations with increasing the distance from the Sun. The minimum values of $n_{\mathrm{c}}$ significantly decrease from 0.4 to $1.0 \mathrm{AU}$ for slow and fast winds, even more than the maximum of $n_{\mathrm{c}}$, resulting in a more significant spread of the data at $1 \mathrm{AU}$ that can also be due to the mix of Cluster and Helios data at this distance. The coefficients of the linear regression $\log _{10}\left(n_{\mathrm{c}}\right)=A V+B$ (where $n$ is the density in $\mathrm{m}^{-3}$ and $V$ is the solar wind velocity in $100 \mathrm{~km} / \mathrm{s}$ to clearly identify the drop in density for $100 \mathrm{~km} / \mathrm{s}$ ) are: $A=-4.06358 \times 10^{-2} \pm 9.52774 \times 10^{-3}$, $B=7.92830$ at $0.4 \mathrm{AU}$, and $A=-2.84225 \times 10^{-2} \pm 1.07845 \times 10^{-2}, B=7.02442$ at 1 AU.

In Figure 2, the halo number density shows a similar anti-correlation, with higher densities in the slow wind. Some of the intermediary slopes of partial decreasing shown by the mean values may be even greater than those obtained for the core. In this case, the coefficients of a similar linear regression with the same units are $A=-2.08976 \times 10^{-1} \pm$ $3.12945 \times 10^{-3}, B=7.03951$ at $0.4 \mathrm{AU}$, and $A=-1.46069 \times 10^{-1} \pm 3.86941 \times 10^{-2}$, $B=6.96072$ at $1 \mathrm{AU}$.

The halo density is lower when the bulk velocity is higher. A high halo density is very important in determining the presence of suprathermal (halo) electrons, even more than (a low) kappa index, in the case of a fit with a sum of two distribution models with different densities and different temperatures, like in the present data set. Indeed, $n_{\mathrm{h}}$ reflects the presence of an additional halo population of energetic electrons, hotter than the core (as shown below), even if the halo would have been Maxwellian (large $\kappa \rightarrow \infty$ ) and not Kappa distributed. The presence of suprathermal particles have a significant effect on the heating of the corona and acceleration of solar wind, whatever they are represented by a sum of two Maxwellians or by Kappa distributions (Pierrard, 2012). Either at low or long distances, the spread of halo densities is large, especially in the slow wind, and decreases in the fast wind. The number density ratio of halo electrons on core electrons vary from 0.3 to $20 \%$ at all radial distances, with an average value decreasing from $6 \%$ for $V<300 \mathrm{~km} / \mathrm{s}$ to $2 \%$ for $V>500 \mathrm{~km} / \mathrm{s}$ at $0.4 \mathrm{AU}$. The same trend is observed at $1 \mathrm{AU}$ : the proportion of halo density remains the same, from 0.4 to $1 \mathrm{AU}$ (Pierrard et al., 2016).

\subsection{Variation of $\kappa$ with the Velocity $V_{\mathrm{SW}}$}

Figure 3 shows the variation of the power-law parameter $\kappa$ with the bulk velocity, left panel at $\approx 0.4 \mathrm{AU}$ and right panel at $\approx 1 \mathrm{AU}$. This parameter quantifies the power-law tail of the distribution function of the halo populations, reproduced in our set of observations by a Kappa function (Štverák et al., 2008). Observational fits with an empirical Kappa impose $\kappa>(l+1) / 2$ to ensure the convergence of the moments of order $l$, which means that we need $\kappa>3 / 2$ to admit a finite temperature and $\kappa>2$ to estimate the heat flux of an anisotropic Kappa (Pierrard and Lemaire, 1996). ${ }^{1}$

At low distances (Helios observations), the mean values of the power parameter $\kappa$ decrease with the solar wind bulk velocity. A similar anti-correlation, but less pronounced, is found for higher distances, i.e. at $1 \mathrm{AU}$ (and $3 \mathrm{AU}$ ). These mean values decrease with increasing the heliocentric distance, but deviations from these means increase with distance,

\footnotetext{
${ }^{1}$ A regularized Kappa distribution with a Maxwellian-like cut-off of the power-law tails ensures the convergence of the moments of any order and restrains to realistic velocities $V<c$, less than the speed of light in vacuum (Lazar et al., 2020).
} 

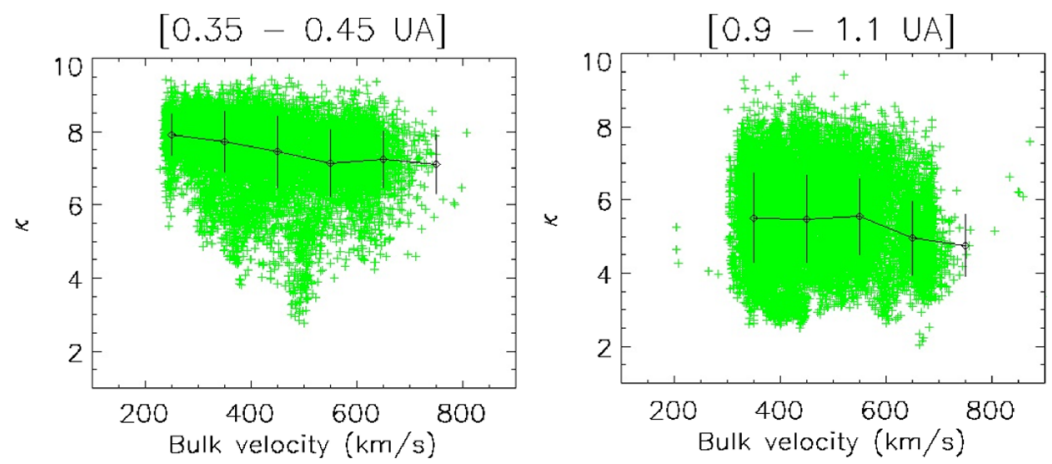

Figure 3 Variation of $\kappa$ with the bulk velocity of SW at $0.35-0.45 \mathrm{AU}$ (left panel) and 0.9-1.1 AU (right panel).
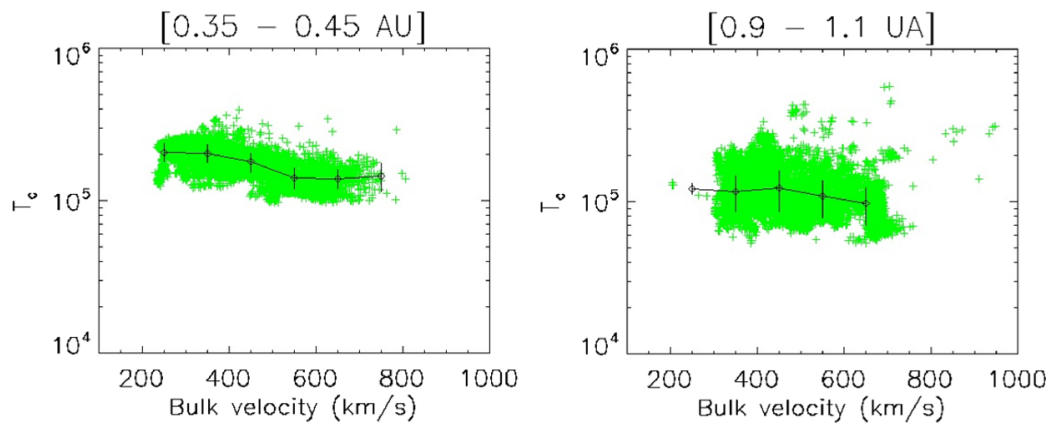

Figure 4 The core temperature $T_{\mathrm{c}}$ (total) in $\mathrm{K}$ as a function of the bulk velocity at $0.35-0.45 \mathrm{AU}$ (left), and $0.9-1.1$ AU (right).

such that values obtained for $\kappa$ at low distances are more concentrated around the means, at $0.4 \mathrm{AU}$ dominant being $6 \leqslant \kappa<9$, while at $1 \mathrm{AU}$ we find $3<\kappa<8$. Similar observations using Kappa models as global representations of electron distributions from Ulysses observations (large distances and at high latitudes) have already shown a lower $\kappa$ may be constantly associated with higher bulk velocities (Maksimovic, Pierrard, and Riley, 1997). Moreover, exospheric solar wind models are able to predict the acceleration of fast streams, provided the existence of suprathermal populations (Pierrard and Lemaire, 1996; Zouganelis et al., 2005).

\subsection{Electron Core and Halo Temperatures}

Figure 4 shows the temperature of the core electrons as a function of the velocity. At low distances, an anti-correlation is observed between the electron temperature and the bulk velocity. It is less clear at larger distances probably due to mix of solar wind particles of different speeds as the solar wind expands. With the new observations of Parker Solar Probe at even lower radial distances ( $0.25 \mathrm{AU})$, this anti-correlation is even more obvious (Maksimovic et al., 2020). This can again be a remnant of coronal hole sources, where electrons of fast wind are observed to be cooler than in the quiet corona. At $0.4 \mathrm{AU}, \log _{10}\left(T_{\mathrm{c}, \|}\right)=a V+b$ (again $V$ in $100 \mathrm{~km} / \mathrm{s}$ and $T$ in $\mathrm{K}$ ) with $a=-3.99357 \times 10^{-2} \pm 4.86351 \times 10^{-4}(\mathrm{~s} / 100 \mathrm{~km})$, 

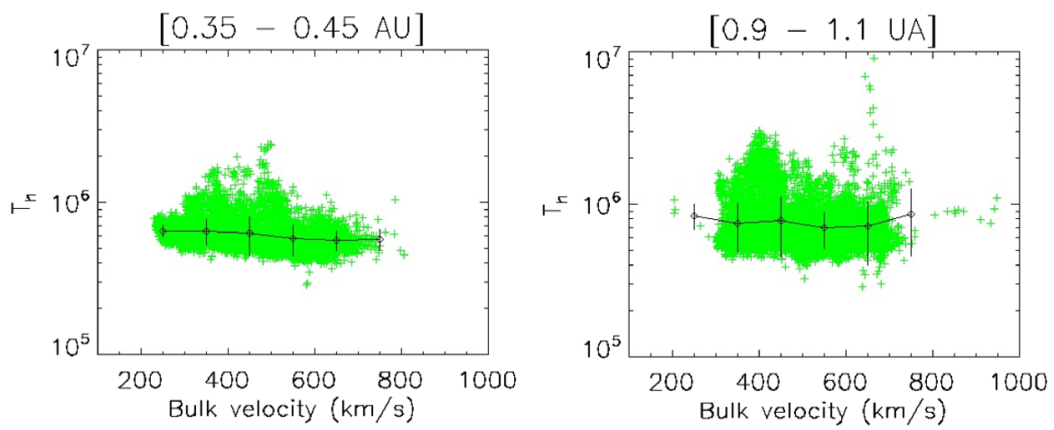

Figure 5 The halo temperature $T_{\mathrm{h}}$ (total) in $\mathrm{K}$ as a function of the bulk velocity at $0.35-0.45 \mathrm{AU}$ (left), and $0.9-1.1 \mathrm{AU}$ (right).

$b=5.44856$ and $\log _{10}\left(T_{\mathrm{c}, \perp}\right)=a V+b$, with $a=-6.89511 \times 10^{-2} \pm 4.73346 \times 10^{-4}$, $b=5.52657$. The anti-correlation is less visible but still present at $1 \mathrm{AU}$, may be due to the mix of Cluster and Helios observations taken during different periods of time and due to the larger distance.

Figure 5 shows the temperature of the halo electrons as a function of the velocity. Parallel and perpendicular temperatures of the halo population are also obtained to be larger in the slow wind. At $0.4 \mathrm{AU}, \log _{10}\left(T_{\mathrm{h}, \|}\right)=a V+b$ with $T$ in $\mathrm{K}$ and $V$ in $100 \mathrm{~km} / \mathrm{s}$, with $a=$ $-1.39837 \times 10^{-2} \pm 6.39593 \times 10^{-4} \mathrm{~s} / 100 \mathrm{~km}, b=5.86102$, and $\log _{10}\left(T_{\mathrm{h}, \perp}\right)=a V+b$, with $a=-2.51076 \times 10^{-2} \pm 5.13766 \times 10^{-4}$, and $b=5.88215$. The bulk velocity is slightly lower for high temperatures whatever it is parallel, perpendicular, core or halo temperature. The slope is low.

Note that this anti-correlation is unexpected, since it is the contrary for the protons, as we will show in Section 3. Moreover, all the models (kinetic or fluid) show a higher bulk velocity when the temperature increases (Pierrard (2009) for a review), at least when all the other parameters are kept the same. Nevertheless, using a lower value of kappa for the strahl for instance, the wind can be accelerated to higher bulk velocities in exospheric solar wind models with Kappa distribution of the electrons, even considering lower temperatures (Pierrard and Lemaire, 1996; Maksimovic, Pierrard, and Lemaire, 1997; Lamy et al., 2003). Concerning possible causes of the speed-temperature anti-correlation, it is worth noting that effects of turbulent heating (Landau damping, cyclotron resonance damping, or stochastic heating), examined in detail for the case of protons, are also acting on electrons, even though both the literature and the observational data are severely lacking. Combination of these mechanisms would provide a similar signature in the data set.

Figure 6 shows the (total) temperature ratio of halo on core electrons as a function of the velocity. The temperature ratio is clearly higher for high velocities, especially at short distances. This is an inverted trend as compared to the individual core and halo temperatures shown in Figures 4 and 5. This indicates that the temperature of the halo decreases less with the velocity than the core temperature.

\subsection{Temperature Anisotropies}

Figures 7 and 8 show the temperature anisotropy $\left(A=T_{\perp} / T_{\|}\right)$of the core and halo electrons, respectively, as a function of the velocity. The temperature anisotropy of the core and halo electrons is reduced (closer to 1) when the bulk velocity is low. Again, this is expected for the core electrons, since the slow wind seems more collisional. The fast wind originates 

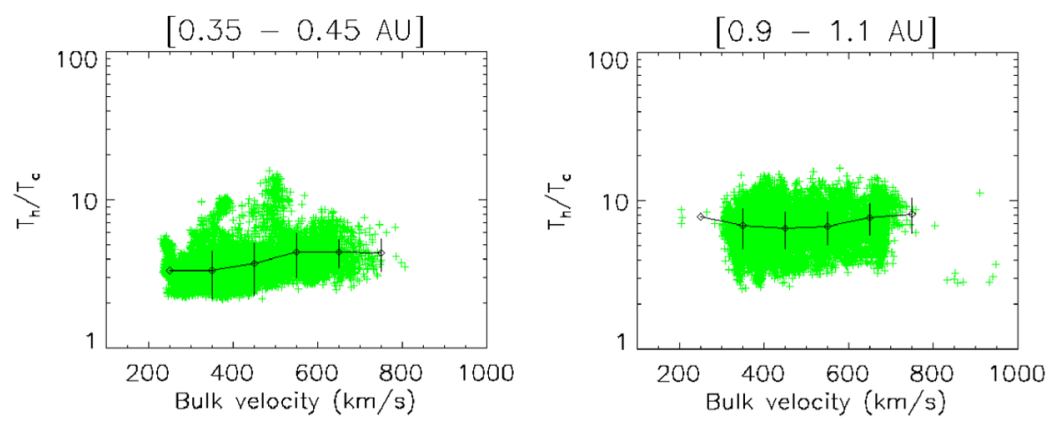

Figure 6 The ratio of halo on core temperatures $T_{\mathrm{h}} / T_{\mathrm{c}}$ (total) as a function of the bulk velocity at $0.35-0.45 \mathrm{AU}$ (left), and 0.9-1.1 AU (right).
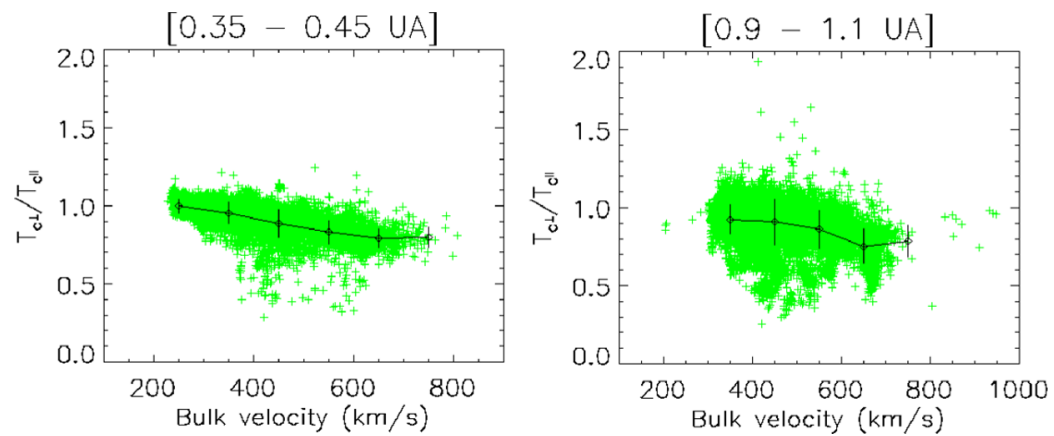

Figure 7 The core temperature anisotropy $T_{\mathrm{c}, \perp} / T_{\mathrm{c}, \|}$ as a function of the bulk velocity at $0.35-0.45 \mathrm{AU}$ (left), and $0.9-1.1 \mathrm{AU}$ (right).

from less dense regions where the collisions are less efficient. The anti-correlation is clear at $0.4 \mathrm{AU}$, especially for the core electrons, and remains visible at $1 \mathrm{AU}$ but with a higher dispersion. The anisotropy slightly decreases with the velocity and is in average $A \approx 0.8$ for the core electrons in the fast wind, thus with $T_{\|}>T_{\perp}$. However the halo electrons are hotter and less dense, and therefore less collisional. In their case the role of binary collisions may be played by the wave-particle collisions, i.e., in the presence of a wave-turbulence, which can entertain their suprathermalization and also prevent large departures from isotropy.

\section{Protons}

Electron and proton densities are almost the same in the solar wind due to the low proportion of heavy ions and the quasi-neutrality. The bulk velocities for electrons and protons are also comparable (although the electron populations, core, halo and strahl, may have relative (opposite) drifts with respect to protons), so that in general no net current is produced (Wilson et al., 2019). The bulk velocity is easier to determine for protons than for electrons, because the thermal velocity $v_{\text {th, }}$ is much lower than for electrons (due to their mass) and is thus well different from the bulk velocity. It is important to keep in mind the different nature of these species: the "local" behavior of protons $\left(v_{\mathrm{th}, \mathrm{p}}<V_{\mathrm{SW}}\right)$ and "non-local" character of electrons $\left(v_{\text {th,e }}>V_{\mathrm{SW}}\right)$, which becomes even more significant for halo compared to core population. 

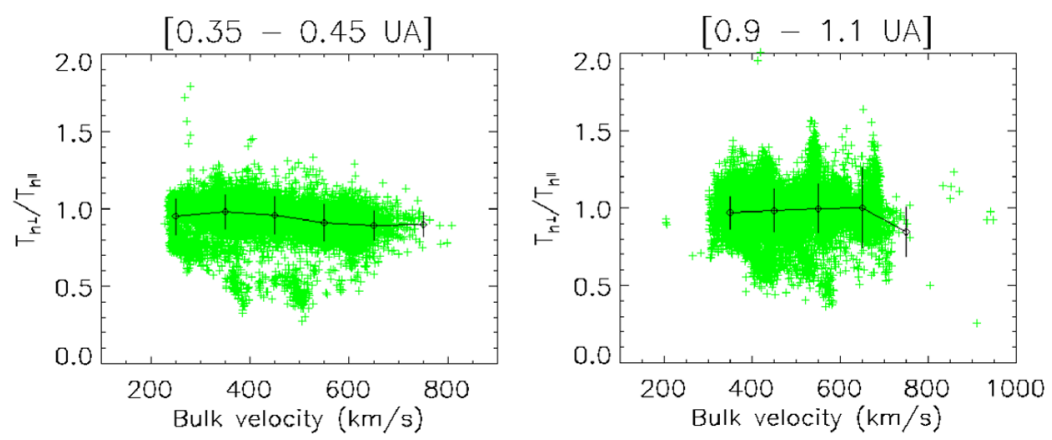

Figure 8 The halo temperature anisotropy $T_{\mathrm{h}, \perp} / T_{\mathrm{h}, \|}$ as a function of the bulk velocity at $0.35-0.45 \mathrm{AU}$ (left), and 0.9-1.1 AU (right).
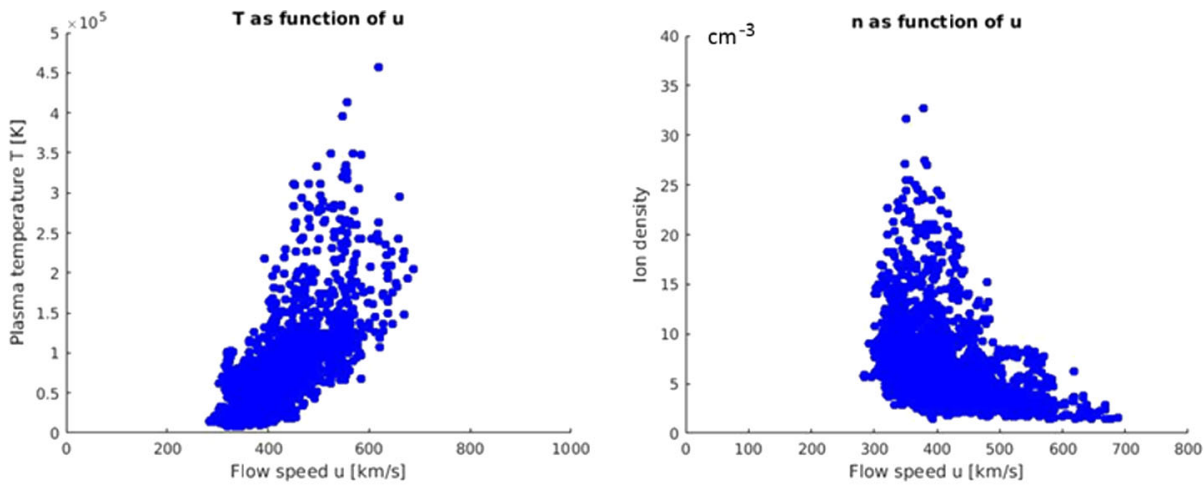

Figure 9 Proton temperature (left) and proton density (right) as a function of the proton bulk velocity at $1 \mathrm{AU}$.

This makes any correlation analysis with some macroscopic characteristics like $V_{\text {SW }}$ easier for protons than for electrons. The temperature of the protons, and also those of the other ions, are very different from the electron temperature (Pierrard and Lamy, 2003) and they have very different relations with the velocity. Helios for instance has also measured proton velocity distribution functions. At $1 \mathrm{AU}$, the density, bulk velocities and proton temperatures as measured by OMNI are available on https://omniweb.gsfc.nasa.gov/.

Figure 9 illustrates the proton temperature (left panel) and proton density (right panel) as a function of the proton bulk velocity at $1 \mathrm{AU}$ (here for instance taking June 2018-October 2018). The proton temperature is clearly directly related to the (proton and electron) bulk velocity of the solar wind. When the proton temperature is higher, the bulk velocity is also higher, as already noted by Burlaga and Ogilvie (1970, 1973). As shown previously (see Figure 4), this is not the case for electrons for which a slight anti-correlation was observed.

High proton bulk velocities are clearly associated to low proton density (like at high latitudes, and like electrons), but here in the ecliptic plane, low-speed wind can be associated to low or high density, probably due to shocks and mix between the slow and fast winds. The shock is produced when fast outflows, like winds or CMEs encounters slow winds, and in the foreshock, the density may increase due to the compression (see Rouillard et al., 2020 for a review). But there are also observations showing lower densities longer after the shock (Richardson et al., 2000). 

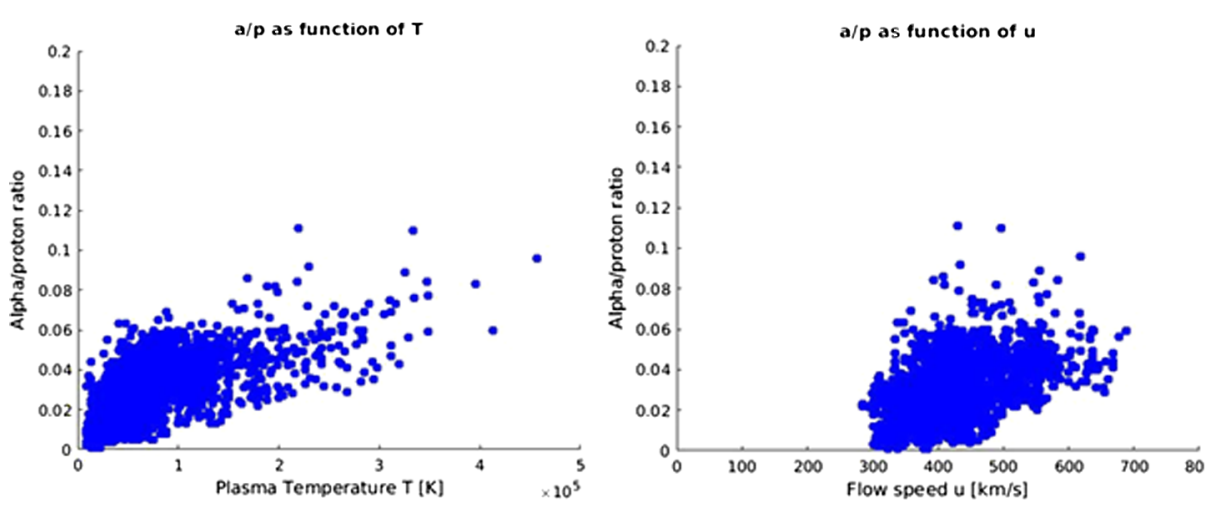

Figure 10 Proportion of helium ions and protons (density ratio), as a function of the solar wind temperature (left panel) and of the proton bulk velocity (right panel) at 1 AU.

\section{Helium Ions}

Figure 10 illustrates the number density ratio of alpha particles on protons as a function of the proton temperature and bulk velocity. Using again OMNI, the proportion of helium ions and protons is found to be highly correlated to the proton temperature and bulk velocity. The proportion of helium ions is higher when the proton temperature is high (Figure 10 left panel), thus also in the high-speed solar wind (right panel) since these two quantities are strongly related. The proportion of helium ions is low (around $5 \%$ ) when the density is high, but for low density, the proportion is variable in the ecliptic plane (Pierrard and Lemaire (2001) for a review).

Kinetic solar wind models also include ions (for instance Pierrard and Pieters (2014)). So the correlations found in the observations for such ions are very useful to make predictions using such models.

\section{Discussion and Conclusions}

Two solar wind types were identified by ULYSSES when flying at different latitudes: the high-speed solar wind associated to low density wind at high latitudes during minimum solar activity, and the low-speed solar wind (low $V$ ) much more variable and associated to high $n$, appearing at low latitudes during minimum solar activity (McComas and et al., 1998).

In the ecliptic plane where the present data are observed, the solar wind velocity continuously varies from slow $(200 \mathrm{~km} / \mathrm{s})$ to fast $(800 \mathrm{~km} / \mathrm{s})$. Strong correlations between the solar wind characteristics can be identified at all radial distances, but are generally much more visible close to the Sun. The velocity is strongly correlated to the temperature of the protons while it is anti-correlated to the temperature of the core and halo electrons. An anticorrelation with the velocity is also obtained for the number density of electrons (of the core and of the halo) and of protons, as well as for the proportion of helium ions. High-speed solar wind is generally associated to low density and low temperature of the electrons, but high temperature of the proton and helium ion proportion. Due to the low density for highspeed solar wind, the collisions are less efficient leading to a lower kappa of the halo and 
temperature anisotropies (for the core and for the halo) further from $A=1$. A low kappa index for the halo (indicating high power-law suprathermal tails in the direction perpendicular to the magnetic field) is related to high-speed solar wind and low number density.

The observations are in good agreement with solar wind models, but the high temperature of the protons associated to low temperature of the electrons is not fully understood. In exospheric models, the presence of energetic electrons accelerates the wind (Pierrard and Pieters, 2014), but it is more the anisotropic strahl population that plays this role than the halo population. The observed halo population (perpendicular to the magnetic field) is more developed in the high-speed wind. The average ratio of suprathermal electrons (halo + strahl) to core density $\left(n_{\mathrm{h}}+n_{\mathrm{st}}\right) / n_{\mathrm{c}}$ remains constant with the distance (Štverák et al., 2009), as the strahl gets scattered into halo, indicating that the population of energetic (suprathermal) electrons is already present at low distances. This ratio of suprathermal on thermal electrons is more important in accelerating the wind than the index kappa, since a sum of two Maxwellians will also lead to an acceleration, due to the higher escaping flux.

The relations and correlations found in the present work are useful to determine links between the parameters to be used in solar wind models. The relations found at low distances can help to determine boundary conditions in solar wind models to make predictions at larger distances. Such results are also significant in determining the evolution of the distribution functions of the particles with the radial distance and better understanding the physical mechanisms implicated in their variations by including them in the models.

Acknowledgements The authors acknowledge useful discussions during the working meetings of the project Kappa Distributions, hosted by the International Space Science Institute (ISSI) in Bern. The authors thank the Belgian Science Policy for the FEDtWIN project ENERGY. These results were obtained in the framework of the projects SCHL 201/35-1 (DFG-German Research Foundation), G0A2316N (FWOVlaanderen).

Declaration of Potential Conflicts of Interest The authors declare they have no conflicts of interest.

Publisher's Note Springer Nature remains neutral with regard to jurisdictional claims in published maps and institutional affiliations.

Open Access This article is licensed under a Creative Commons Attribution 4.0 International License, which permits use, sharing, adaptation, distribution and reproduction in any medium or format, as long as you give appropriate credit to the original author(s) and the source, provide a link to the Creative Commons licence, and indicate if changes were made. The images or other third party material in this article are included in the article's Creative Commons licence, unless indicated otherwise in a credit line to the material. If material is not included in the article's Creative Commons licence and your intended use is not permitted by statutory regulation or exceeds the permitted use, you will need to obtain permission directly from the copyright holder. To view a copy of this licence, visit http://creativecommons.org/licenses/by/4.0/.

\section{References}

Berčič, L., Maksimović, M., Landi, S., Matteini, L.: 2019, Scattering of strahl electrons in the solar wind between 0.3 and 1 au: Helios observations. Mon. Not. Roy. Astron. Soc. 486, 3404. DOI.

Berčič, L., Larson, D., Whittlesey, P., Maksimović, M., Badman, S.T., Landi, S., Matteini, L., Bale, S.D., Bonnell, J.W., Case, A.W., de Wit, T.D., Goetz, K., Harvey, P.R., Kasper, J.C., Korreck, K.E., Livi, R., MacDowall, R.J., Malaspina, D.M., Pulupa, M., Stevens, M.L.: 2020, Coronal electron temperature inferred from the strahl electrons in the inner heliosphere: Parker solar probe and Helios observations. Astrophys. J. 892, 88. DOI.

Burlaga, L.F., Ogilvie, K.W.: 1970, Heating of the solar wind. Astrophys. J. 159, 659. DOI.

Burlaga, L.F., Ogilvie, K.W.: 1973, Solar wind temperature and speed. J. Geophys. Res. 78, 2028. DOI.

Domingo, V.: 2002, SOHO, Yohkoh, Ulysses and Trace: the four solar missions in perspective, and available resources. Astrophys. Space Sci. 282, 171. DOI. 
Ďurovcová, T., Šafránková, J., Němeček, Z.: 2019, Evolution of relative drifts in the expanding solar wind: Helios observations. Solar Phys. 294, 97. DOI.

Elliott, H.A., Henney, C.J., McComas, D.J., Smith, C.W., Vasquez, B.J.: 2012, Temporal and radial variation of the solar wind temperature-speed relationship. J. Geophys. Res. Space Phys. 117, A09102. DOI.

Gloeckler, G., Zurbuchen, T.H., Geiss, J.: 2003, Implications of the observed anticorrelation between solar wind speed and coronal electron temperature. J. Geophys. Res. Space Phys. 108, 158. DOI.

Halekas, J.S., Whittlesey, P., Larson, D.E., McGinnis, D., Maksimovic, M., Berthomier, M., Kasper, J.C., Case, A.W., Korreck, K.E., Stevens, M.L., Klein, K.G., Bale, S.D., MacDowall, R.J., Pulupa, M.P., Malaspina, D.M., Goetz, K., Harvey, P.R.: 2020, Electrons in the young solar wind: first results from the parker solar probe. Astrophys. J. Suppl. 246, 22. DOI.

Hammond, C.M., Feldman, W.C., McComas, D.J., Phillips, J.L., Forsyth, R.J.: 1996, Variation of electronstrahl width in the high-speed solar wind: Ulysses observations. Astron. Astrophys. 316, 350.

Klein, K.G., Martinovic, M., Stansby, D., Horbury, T.S.: 2019, Linear stability in the inner heliosphere: Helios re-evaluated. Astrophys. J. 887, 234. DOI.

Lamy, H., Pierrard, V., Maksimovic, M., Lemaire, J.: 2003, A kinetic exospheric model of the solar wind with a non monotonic potential energy for the protons. J. Geophys. Res. 108, 1047. DOI.

Lazar, M. (ed.): 2012, Exploring the Solar Wind, IntechOpen, Rijeka. DOI.

Lazar, M., Pierrard, V., Shaaban, S., Fichtner, H., Poedts, S.: 2017, Dual Maxwellian-kappa modeling of the solar wind electrons: new clues on the temperature of kappa populations. Astron. Astrophys. 602, A44. DOI.

Lazar, M., López, R.A., Shaaban, S.M., Poedts, S., Fichtner, H.: 2019, Whistler instability stimulated by the suprathermal electrons present in space plasmas. Astrophys. Space Sci. 364, 171. DOI.

Lazar, M., Scherer, K., Fichtner, H., Pierrard, V.: 2020, Toward a realistic macroscopic parametrization of space plasmas with regularized $\kappa$-distributions. Astron. Astrophys. 634, A20. DOI.

Maksimovic, M., Pierrard, V., Lemaire, J.: 1997, A kinetic model of the solar wind with Kappa distributions in the corona. Astron. Astrophys. 324, 725.

Maksimovic, M., Pierrard, V., Riley, P.: 1997, Ulysses electron distributions fitted with Kappa functions. Geophys. Res. Lett. 24, 1151.

Maksimovic, M., Zouganelis, I., Chaufray, J.-Y., Issautier, K., Scime, E., Littleton, J., Marsch, E., McComas, D., Salem, C., Lin, R., et al.: 2005, Radial evolution of the electron distribution functions in the fast solar wind between 0.3 and 1.5 au. J. Geophys. Res. 110, A09104. DOI.

Maksimovic, M., Bale, S.D., Berčič, L., Bonnell, J.W., Case, A.W., Wit, T.D.d., Goetz, K., Halekas, J.S., Harvey, P.R., Issautier, K., Kasper, J.C., Korreck, K.E., Jagarlamudi, V.K., Lahmiti, N., Larson, D.E., Lecacheux, A., Livi, R., MacDowall, R.J., Malaspina, D.M., Martinović, M.M., Meyer-Vernet, N., Moncuquet, M., Pulupa, M., Salem, C., Stevens, M.L., Štverák, Š., Velli, M., Whittlesey, P.L.: 2020, Anticorrelation between the bulk speed and the electron temperature in the pristine solar wind: first results from the Parker solar probe and comparison with Helios. Astrophys. Suppl. Ser. 246, 62. DOI.

Matteini, L., Landi, S., Hellinger, P., Pantellini, F., Maksimovic, M., Velli, M., Goldstein, B.E., Marsch, E.: 2007, Evolution of the solar wind proton temperature anisotropy from 0.3 to 2.5 au. Geophys. Res. Lett. 34, L20105. DOI.

McComas, D.J., et al.: 1998, Ulysses' return to the slow solar wind. Geophys. Res. Lett. 25, 1. DOI.

Meyer-Vernet, N.: 2007, Basics of the Solar Wind, Cambridge Atmospheric and Space Science Series, Cambridge University Press, Cambridge. DOI.

Moschou, S.P., Pierrard, V., Keppens, R., Pomoell, J.: 2017, Interfacing MHD and kinetic solar wind models and comparing their energetics. Solar Phys. 292, 1. DOI.

Pierrard, V.: 2009, L'environnement spatial de la Terre, Presses Universitaires de Louvain, Louvain-LaNeuve. 978-2-87463-195-5.

Pierrard, V.: 2012, Effects of suprathermal particles in space plasmas. In ICNS Annual International Astrophysics Conference Proc. 1436, 61. DOI.

Pierrard, V., Lamy, H.: 2003, The effects of the velocity filtration mechanism on the minor ions of the corona. Solar Phys. 216, 47. DOI.

Pierrard, V., Lazar, M.: 2010, Kappa distributions: theory and applications in space plasmas. Solar Phys. 267, 153. DOI.

Pierrard, V., Lazar, M., Schlickeiser, R.: 2011, Evolution of the electron distribution function in the wave turbulence of the solar wind. Solar Phys. 269, 421. DOI.

Pierrard, V., Lemaire, J.: 1996, Lorentzian ion exosphere model. J. Geophys. Res. 103, 7923. DOI.

Pierrard, V., Lemaire, J.: 2001, Kinetic models of solar and polar winds. Astrophys. Space Sci. 277, 169. DOI.

Pierrard, V., Maksimovic, M., Lemaire, J.: 2001a, Core, halo and strahl electrons in the solar wind. Astrophys. Space Sci. 277, 195. DOI.

Pierrard, V., Maksimovic, M., Lemaire, J.: 2001b, Self-consistent model of solar wind electrons. J. Geophys. Res. 106, 29305. DOI. 
Pierrard, V., Pieters, M.: 2014, Coronal heating and solar wind acceleration for electrons, protons and minor ions obtained from kinetic models based on Kappa distributions. J. Geophys. Res. 119, 9441. DOI.

Pierrard, V., Lazar, M., Poedts, S., Štverák, Š., Maksimovic, M., Trávníček, P.: 2016, The electron temperature and anisotropy in the solar wind. Comparison of the core and halo populations. Solar Phys. 291, 2165. DOI.

Pomoell, J., Poedts, S.: 2018, EUHFORIA: European heliospheric forecasting information asset. J. Space Weather Space Clim. 14, A35. DOI.

Richardson, I.G., Berdichevsky, D., Desch, M.D., Farrugia, C.J.: 2000, Solar-cycle variation of low density solar wind during more than three solar cycles. Geophys. Res. Lett. 27, 3761. DOI.

Rouillard, A., Viall, N., Vocks, C., Wu, Y., Pinto, R., Lavarra, M., Matteini, L., Pierrard, V., Sanchez-Diaz, E., Alexandrova, O., Lavraud, B.: 2020, Solar Wind. In AGU book.

Scudder, J.D.: 1992, On the causes of temperature change in inhomogeneous low-density astrophysical plasmas. Astrophys. J. 398, 299. DOI.

Stansby, D., Horbury, T.S., Matteini, L.: 2019, Diagnosing solar wind origins using in situ measurements in the inner heliosphere. Mon. Not. Roy. Astron. Soc. Lett. 482, 1706. DOI.

Stansby, D., Salem, C., Matteini, L., Horbury, T.: 2018, A new inner heliosphere proton parameter dataset from the Helios mission. Solar Phys. 293, 155. DOI.

Štverák, Š. ., Trávníček, P.M., Hellinger, P.: 2015, Electron energetics in the expanding solar wind via Helios observations. J. Geophys. Res. 120, 8177. DOI.

Štverák, Š., Trávníček, P., Maksimovic, M., Marsch, E., Fazakerley, A.N., Scime, E.E.: 2008, Electron temperature anisotropy constraints in the solar wind. J. Geophys. Res. 113, A03103. DOI.

Štverák, Š., Maksimovic, M., Trávníček, P., Marsch, E., Fazakerley, A.N., Scime, E.E.: 2009, Radial evolution of nonthermal electron populations in the low-latitude solar wind: Helios, Cluster, and Ulysses observations. J. Geophys. Res. 114, A05104. DOI.

Vocks, C., Mann, G.: 2003, Generation of suprathermal electrons by resonant wave-particle interaction in the solar corona and wind. Astrophys. J. 593, 1134. ADS.

Vocks, C., Salem, C., Lin, R.P., Mann, G.: 2005, Electron halo and strahl formation in the solar wind by resonant interaction with whistler waves. Astrophys. J. 627, 540.

Wilson, L.B., Koval, A., Szabo, A., Breneman, A., Cattell, C.A., Goetz, K., Kellogg, P.J., Kersten, K., Kasper, J.C., Maruca, B.A., Pulupa, M.: 2013, Electromagnetic waves and electron anisotropies downstream of supercritical interplanetary shocks. J. Geophys. Res. 118, 5. DOI.

Wilson, L.B., Chen, L.-J., Wang, S., Schwartz, S.J., Turner, D.L., Stevens, M.L., Kasper, J.C., Osmane, A., Caprioli, D., Bale, S.D., Pulupa, M.P., Salem, C.S., Goodrich, K.A.: 2019, Electron energy partition across interplanetary shocks. II. Statistics. Astrophys. J. Suppl. 245, 24. DOI.

Zouganelis, I., Meyer-Vernet, N., Landi, S., Maksimovic, M., Pantellini, F.: 2005, Acceleration of weakly collisional solar-type winds. Astrophys. J. Lett. 626, 1151. 